TRANSACTIONS OF THE

AMERICAN MATHEMATICAL SOCIETY

Volume 348, Number 7, July 1996

\title{
LOW CODIMENSIONAL SUBMANIFOLDS OF EUCLIDEAN SPACE WITH NONNEGATIVE ISOTROPIC CURVATURE
}

\author{
FRANCESCO MERCURI AND MARIA HELENA NORONHA
}

\begin{abstract}
In this paper we study compact submanifolds of Euclidean space with nonnegative isotropic curvature and low codimension. We determine their homology completely in the case of hypersurfaces and for some low codimensional conformally flat immersions.
\end{abstract}

\section{INTRODUCTION}

In [13], Micallef and Moore introduced the concept of curvature on totally isotropic two-planes for manifolds of dimension $\geq 4$. We will call it, for brevity, isotropic curvature. This curvature plays a similar role in the study of stability of harmonic 2-spheres that the sectional curvature does in the study of stability of geodesics. In fact, studying the index of harmonic 2-spheres in a compact Riemannian manifold $M$, Micallef and Moore proved that if $M$ has positive isotropic curvature then the homotopy groups $\pi_{i}(M)$ vanish for $2 \leq i \leq[n / 2]$. Therefore, if $\pi_{1}(M)$ is finite then the Betti numbers $\beta_{i}(M)$ are zero for $2 \leq i \leq n-2$.

There exists a topological classification in dimension 4 for compact manifolds with nonnegative isotropic curvature. ( [19] and [14] Theorem 4.10 ). If such a manifold is simply connected then it is either homeomorphic to a sphere, or biholomorphic to the complex projective space $\mathbf{C P}^{2}$ or is a product of two surfaces where one of them is homeomorphic to a sphere. For higher dimensions, the only known results appear in [14] and [20] and some of them will be discussed in this article.

The main purpose of this paper is to study compact submanifolds of Euclidean space with nonnegative isotropic curvature and whose codimension is sufficiently low. Our first result describes the topology of hypersurfaces.

Theorem 1. Let $f: M^{n} \rightarrow \mathbf{R}^{n+1}, n \geq 4$, be an isometric immersion of a compact manifold $M$ with nonnegative isotropic curvature. Then the homology groups $H_{i}(M ; \mathbf{Z})=0$ for $2 \leq i \leq n-2$ and the fundamental group $\pi_{1}(M)$ is a free group on $\beta_{1}$ elements.

In [14], considering connected sums of compact manifolds with positive isotropic curvature, they show that the fundamental group of a compact manifold with positive isotropic curvature can be very large. We show that this can happen also in the

Received by the editors March 24, 1995.

1991 Mathematics Subject Classification. Primary 53C40, 53C42.

Key words and phrases. Isotropic curvature, Betti numbers, hypersurfaces, conformally flat manifolds.

The first author's research was partially supported by CNPq, Brasil.

(C)1996 American Mathematical Society 
case of hypersurfaces of nonnegative isotropic curvature. Actually, we show that given a nonnegative number $\beta_{1}$, we can construct a compact hypersurface embedded in $\mathbf{R}^{n+1}$ with nonnegative isotropic curvature whose $\pi_{1}(M)$ is a free group generated by $\beta_{1}$ elements. This hypersurface is a conformally flat manifold and the same type of construction furnishes examples of compact conformally flat hypersurfaces of dimension greater than 4 that have nonnegative scalar curvature but some negative isotropic curvature, showing that the equivalence between the nonnegativity of these two curvatures is true for conformally flat manifolds if and only if the dimension is four. Of course this fact can be easily seen away from the context of hypersurfaces. For instance, a Riemannian product $H^{k} \times S^{k}$ for $k \geq 3$, where $H^{k}$ is a hyperbolic space and $S^{k}$ is a sphere with its standard metric is conformally flat, has scalar curvature identically zero and some isotropic curvatures that are negative. In [14], they observed that $\Sigma_{g} \times S^{2 k}$, where $\Sigma_{g}$ is a Riemann surface of genus $g \geq 2$, and $k \geq 2$, admits a conformally flat metric with positive scalar curvature but admits no metric with positive isotropic curvature. However, this manifold does admit a conformally flat metric with nonnegative isotropic curvature and in fact, we show that the only locally reducible conformally flat manifolds with nonnegative isotropic curvature are flat or covered by the product of a hyperbolic plane with a sphere.

For conformally flat manifolds we prove the following result.

Theorem 2. Let $M^{n}, n \geq 4$, be a compact conformally flat manifold with nonnegative isotropic curvature. Then either $M$ is flat or $\beta_{i}(M)=0$ for $3 \leq i \leq n-3$. Moreover if $\beta_{2}(M) \neq 0$ then either $M$ is flat or it is isometrically covered by the product of a hyperbolic plane with an $(n-2)$-sphere with its standard metric.

Using the results in [16] on isometric immersions of conformally flat manifolds of Euclidean space, we deduce the following corollary.

Corollary 1. Let $f: M^{n} \rightarrow \mathbf{R}^{n+p}, 2 \leq p \leq n / 2-1$, be an isometric immersion of a compact, orientable conformally flat manifold $M$ with nonnegative isotropic curvature. Then $H_{i}(M ; \mathbf{Z})=0$ for $p \leq i \leq n-p$.

We prove these results in section 4 . In the same section, we extend the results of Theorem 2.1 in [14] for odd dimensional manifolds whose curvature tensor is pure (see Definition 4.5). We prove in this case that if $M$ is a locally irreducible odd dimensional manifold then $\beta_{2}(M)=0$.

It is a simple but interesting fact to observe that the product of two manifolds of nonnegative isotropic curvature may not have nonnegative isotropic curvature. A rather complete description of locally reducible compact manifolds with nonnegative isotropic curvature is given in [14]. The structure of locally reducible codimension 2 submanifolds with nonnegative isotropic curvature is described by the following:

Theorem 3. Let $f: M^{n} \rightarrow \mathbf{R}^{n+2}, n \geq 4$, be an isometric immersion of a compact, locally reducible manifold $M$ with nonnegative isotropic curvature. Then one of the following holds:

i) $M$ has nonnegative sectional curvature and is either the total space of a sphere bundle over $S^{1}$ or $f$ is the product of two convex embeddings.

ii) There exists an isometric covering $\pi: M_{1} \times \Sigma^{2} \rightarrow M$, where $\Sigma^{2}$ is a surface and $\left.(f \circ \pi)\right|_{M_{1} \times\{p\}}$ is a convex embedding into some $(n-1)$-dimensional affine subspace of $\mathbf{R}^{n+2}$.

It follows from Theorem 2 in [5] that the other cases of codimension 2 submanifolds are those whose holonomy algebra is either the unitary algebra $u(2)$ and the 
manifold is four dimensional or the exterior algebra $o(n)$. We also study these cases. In the former case, we obtain that the second Betti number $\beta_{2}(M)=0$ and $M$ is covered by a noncompact Kähler manifold. For the latter case we assume pure curvature tensor, and using the results of section 4, we conclude then that $\beta_{2}(M)=0$.

Part of the research of this article was done when the second author visited the Universidade Estadual de Campinas in Brasil. She wants to thank the Department of Mathematics for the hospitality and the state agency FAPESP for the financial support.

\section{NotATIONS AND BASIC FACTS}

In what follows $M$ will always denote an n-dimensional Riemannian manifold with $n \geq 4$. For $x$ in $M$ we consider the complexified tangent space $T_{x} M \otimes \mathbf{C}$ and we extend the Riemannian metric $\langle$,$\rangle to a complex bilinear form ($,$) . An element$ $Z$ in $T_{x} M \otimes \mathbf{C}$ is said to be isotropic if $(Z, Z)=0$. A two plane $\sigma \subset T_{x} M \otimes \mathbf{C}$ is totally isotropic if $(Z, Z)=0$ for any $Z \in T_{x} M \otimes \mathbf{C}$. If $\sigma$ is a totally isotropic two-plane then there exists a basis $\{Z, W\}$ of $\sigma$ such that

$$
Z=e_{i}+\sqrt{-1} e_{j} \text { and } W=e_{k}+\sqrt{-1} e_{m}
$$

where $e_{i}, e_{j}, e_{m}, e_{k}$ are orthonormal vectors of $T_{x} M$. Conversely, any two such vectors span a totally isotropic two plane. Let $\widetilde{\mathcal{R}}$ denote the complex linear extension of the curvature operator $\mathcal{R}: \Lambda^{2} \rightarrow \Lambda^{2}$.

Definition 2.1. A Riemannian manifold is said to have nonnegative isotropic curvature if $\langle\widetilde{\mathcal{R}}(Z \wedge W),(Z \wedge W)\rangle \geq 0$ whenever $\{Z, W\}$ is a totally isotropic two plane.

It follows from this definition that for $Z$ and $W$ as above

$$
\langle\widetilde{\mathcal{R}}(Z \wedge W),(Z \wedge W)\rangle=K_{i k}+K_{i m}+K_{j k}+K_{j m}-2\left\langle R\left(e_{i}, e_{j}\right) e_{k}, e_{m}\right\rangle \geq 0
$$

where $K_{i k}$ denotes the sectional curvature of the plane spanned by $e_{i}, e_{k}$ and $R$ is the curvature tensor of $M$ (see [13] p.203). If we consider $\bar{Z}=e_{i}-\sqrt{-1} e_{j}$, then $\{\bar{Z}, W\}$ is a totally isotropic two plane. Performing the same computation for $\langle\widetilde{\mathcal{R}}(\bar{Z} \wedge W),(\bar{Z} \wedge W)\rangle$ and adding with $\langle\widetilde{\mathcal{R}}(Z \wedge W),(Z \wedge W)\rangle$ we get

Lemma 2.2. Let $M$ be a manifold with nonnegative isotropic curvature. Then for all sets of orthonormal vectors $e_{i}, e_{j}, e_{m}, e_{k}$ in $T_{x} M$ we have

$$
K_{i k}+K_{i m}+K_{j k}+K_{j m} \geq 0
$$

Remark 2.3. a) The condition of Lemma 2.2 is not sufficient for the nonnegativity of the isotropic curvature. However, if $M$ is conformally flat, $\left\langle R\left(e_{i}, e_{j}\right) e_{k}, e_{m}\right\rangle=0$ for all orthonormal vectors and therefore, in this case, this condition is equivalent to nonnegative isotropic curvature.

b) Nonnegative isotropic curvature implies nonnegative scalar curvature ( see [14] Proposition 2.5). If $M$ is conformally flat and four-dimensional, the nonnegativity of the scalar curvature implies the nonnegativity of the isotropic curvature. But, if the dimension is at least 5, this is no longer true, even for hypersurfaces of Euclidean spaces ( see section 3 ). 
In this paper we will be mostly interested in the case where $M$ is isometrically immersed in a Euclidean space. For an isometric immersion $f: M^{n} \rightarrow \mathbf{R}^{n+p}$ we will use the following notation: $\nu(M)$ will be the normal bundle of the immersion and $\alpha: T M \oplus T M \rightarrow \nu(M)$ the second fundamental form; $H=\frac{1}{n}$ trace $\alpha$ is the mean curvature vector and $N(x)=\left\{X \in T_{x} M \mid \alpha(X, Y)=0, \forall Y \in T_{x} M\right\}$ the relative nullity space. If $\xi \in \nu_{x} M$, we will denote by $A_{\xi}$ the Weingarten operator given by $\left\langle\left\langle A_{\xi}(X), Y\right\rangle=\langle\alpha(X, Y), \xi\rangle\right.$. Recall that the second fundamental form is related to the curvature tensor $R$ by means of the Gauss equation

$$
\langle R(X, Y) Z, W\rangle=\langle\alpha(X, W), \alpha(Y, Z)\rangle-\langle\alpha(X, Z), \alpha(Y, W)\rangle .
$$

In the next proposition we show some properties of the second fundamental form of an isometric immersion of manifolds with nonnegative isotropic curvature.

Proposition 2.4. Let $f: M^{n} \longrightarrow \mathbf{R}^{n+p}$ be an isometric immersion with second fundamental form $\alpha$ and mean curvature vector $H$. If $M$ has nonnegative isotropic curvature and $e_{i}, e_{j}, e_{m}, e_{k}$ are orthonormal vectors of $T_{x}(M)$ then

(a) $\left\langle\alpha\left(e_{i}, e_{i}\right)+\alpha\left(e_{j}, e_{j}\right), \alpha\left(e_{k}, e_{k}\right)+\alpha\left(e_{m}, e_{m}\right)\right\rangle \geq 0$,

(b) $\left\langle\alpha\left(e_{i}, e_{i}\right)+\alpha\left(e_{j}, e_{j}\right), H\right\rangle \geq 0$,

(c) If $\operatorname{dim} N(x)=0$, then $H(x) \neq 0$,

(d) If $\operatorname{dim} N(x) \neq 0$, then the Ricci curvatures at $x$ are nonnegative.

Proof. Applying Lemma 2.2 to the Gauss equation we get

$$
\begin{aligned}
& \left\langle\alpha\left(e_{i}, e_{i}\right), \alpha\left(e_{k}, e_{k}\right)\right\rangle+\left\langle\alpha\left(e_{i}, e_{i}\right), \alpha\left(e_{m}, e_{m}\right)\right\rangle \\
& \quad+\left\langle\alpha\left(e_{j}, e_{j}\right), \alpha\left(e_{k}, e_{k}\right)\right\rangle+\left\langle\alpha\left(e_{j}, e_{j}\right), \alpha\left(e_{m}, e_{m}\right)\right\rangle \\
& \quad \geq\left\|\alpha\left(e_{i}, e_{k}\right)\right\|^{2}+\left\|\alpha\left(e_{i}, e_{m}\right)\right\|^{2}+\left\|\alpha\left(e_{j}, e_{k}\right)\right\|^{2}+\left\|\alpha\left(e_{i}, e_{m}\right)\right\|^{2} \geq 0
\end{aligned}
$$

which implies (a). Now if the dimension of the manifold $n$ is even, then (a) implies (b). If $n$ is odd, we observe that given orthonormal vectors $e_{i}$ and $e_{j}$ there exists $e_{m}$ such that $K_{i m}+K_{j m} \geq 0$; otherwise we would have $K_{i k}+K_{i m}+K_{j k}+K_{j m}<0$ for all $e_{k}$ and $e_{m}$ orthogonal to $e_{i}$ and $e_{j}$, contradicting Lemma 2.2. Therefore, from the Gauss equation we get

$$
\left\langle\alpha\left(e_{i}, e_{i}\right)+\alpha\left(e_{j}, e_{j}\right), \alpha\left(e_{m}, e_{m}\right)\right\rangle \geq\left\|\alpha\left(e_{i}, e_{m}\right)\right\|^{2}+\left\|\alpha\left(e_{j}, e_{m}\right)\right\|^{2} \geq 0
$$

that together with (a) for $n$ odd, gives (b). In order to obtain (c) suppose that $H(x)=0$ and $\operatorname{dim} N(x)=0$. Consider $\left\{e_{1}, \ldots, e_{n}\right\}$ an orthonormal basis of the tangent space. Therefore $\left\langle\alpha\left(e_{i}, e_{i}\right)+\alpha\left(e_{j}, e_{j}\right), H\right\rangle=0$ for every $i \neq j$. Since the left hand side of this equation is a sum of nonnegative numbers, it follows then that $\alpha\left(e_{i}, e_{i}\right)+\alpha\left(e_{j}, e_{j}\right)=0$ for every $i \neq j$. But this implies that $\alpha\left(e_{j}, e_{j}\right)=0$ for every $e_{j}$. Then from the Gauss equation we get that the sectional curvatures $K_{i j}$ are given by $K_{i j}=-\left\|\alpha\left(e_{i}, e_{j}\right)\right\|^{2}$ for every $i \neq j$. This in Lemma 2.2 gives $\alpha\left(e_{i}, e_{j}\right)=0$ for every $i \neq j$, contradicting that $\operatorname{dim} N(x)=0$. To prove (d), suppose that $e_{1} \in N(x)$. Then $\operatorname{Ric}\left(e_{1}, e_{j}\right)=0$ for every $j$ and $e_{1}$ is an eigenvector of the symmetric curvature Ricci operator that will be denoted by Ric. Let $\left\{e_{1}, \ldots, e_{n}\right\}$ be an orthonormal basis of eigenvectors of Ric. If $\operatorname{Ric}\left(e_{i}\right)<0$ there exist $e_{j}, e_{k}$ such that $K_{i j}+K_{i k}<0$. Therefore $e_{1}, e_{i}, e_{j}, e_{k}$ are orthonormal vectors and $K_{1 j}+K_{1 k}+K_{i j}+K_{i k}<0$ contradicts again Lemma 2.2 .

\section{Hypersurfaces With NONNEGative isotropic CURVATURE}

Proof of Theorem 1. Let $f: M^{n} \longrightarrow \mathbf{R}^{n+1}$ be an isometric immersion of a compact, connected manifold $M$. Let $\xi$ be a unit vector such that $\pm \xi$ are regular values 
of the Gauss map $\Phi: M^{n} \rightarrow S^{n} \subseteq \mathbf{R}^{n+1}$. Then the height function $h_{\xi}: M \rightarrow \mathbf{R}$ given by $h_{\xi}(x)=<f(x), \xi>$ is a Morse fuction with critical points $\Phi^{-1}( \pm \xi)$. At such points the hessian of $h_{\xi}$ is given, up to a sign, by the Weingarten operator $A_{\xi}$. Let $\left\{e_{1}, \ldots, e_{n}\right\}$ be an orthonormal basis of $T_{x} M$ that diagonalizes $A_{\xi}$, say, $A_{\xi} e_{i}=\lambda_{i} e_{i}$. By the Gauss equation $K_{i j}=\lambda_{i} \lambda_{j}$ and since the critical points are non-degenerate, we have that $\lambda_{i} \neq 0$ for $i=1, \ldots, n$. Therefore if $M$ has nonnegative isotropic curvature Lemma 2.2 implies

$$
\left(\lambda_{i}+\lambda_{j}\right)\left(\lambda_{k}+\lambda_{m}\right) \geq 0
$$

for all distinct indices $i, j, k, m$. Then we conclude that all but at most one of the $\lambda_{i}^{\prime} s$ have the same sign and hence the index of a critical point of $h_{\xi}$ has to be $0,1, n-1$ or $n$. By the standard Morse Theory, $M$ has the homotopy type of a $C W$-complex, with no cells of dimension $i$ for $2 \leq i \leq n-2$. Therefore the homology group $H_{i}(M ; \mathbf{Z})=0$ for $2 \leq i \leq n-2$. Moreover, since there are no 2 -cells $(n \geq 4)$, we conclude by the cellular aproximation theorem that the inclusion of the 1-skeleton $M^{(1)} \hookrightarrow M$ induces an isomorphism between the fundamental groups. Therefore the fundamental group $\pi_{1}(M)$ is a free group on $\beta_{1}$ elements and $H_{1}(M ; \mathbf{Z})$ is a free abelian group with the same number of generators.

Theorem 1 shows that the topology of compact hypersurfaces of nonnegative isotropic curvature is very similar to the topology of compact conformally flat hypersurfaces ( see [8] ). Therefore we look for examples in this class of hypersurfaces. Conformally flat hypersurfaces of $\mathbf{R}^{n+1}, n \geq 4$, are characterized by the fact that the Weingarten operator $A_{\xi}$ has an eigenvalue of multiplicity at least $(n-1)$, i.e., they are almost umbilic ( see [8] for instance). In the next proposition we give a necessary and sufficient condition for the nonnegativity of the isotropic curvature of a conformally flat hypersurface of Euclidean space.

Proposition 3.1. Let $f: M^{n} \longrightarrow \mathbf{R}^{n+1}, n \geq 4$, be an almost umbilic isometric immersion. Let $\lambda$ denote the eigenvalue of multiplicity $(n-1)$ and $\mu$ be the other eigenvalue. Let us suppose that the Weingarten operator is oriented such that $\lambda \geq 0$. Then $M$ has nonnegative isotropic curvature if and only if $\lambda+\mu \geq 0$.

Proof. Let $\left\{e_{1}, \ldots, e_{n}\right\}$ be an orthonormal basis of $T_{x} M$ that diagonalizes $A_{\xi}$ such that $e_{1}$ is the eigenvector correponding to the eigenvalue $\mu$. If $\{Z, W\}$ is a totally isotropic two plane such that

$$
Z=e_{i}+\sqrt{-1} e_{j} \text { and } W=e_{m}+\sqrt{-1} e_{k}
$$

then $\langle\widetilde{\mathcal{R}}(Z \wedge W),(Z \wedge W)\rangle$ is either $2 \lambda(\lambda+\mu)$ or $4 \lambda^{2}$. Therefore if $M$ has nonnegative isotropic curvature we have $\lambda+\mu \geq 0$. Conversely, consider a totally isotropic two plane $\{Z, W\}$ such that

$$
Z=X_{1}+\sqrt{-1} X_{2} \text { and } W=X_{3}+\sqrt{-1} X_{4}
$$

where $X_{1}, X_{2}, X_{3}, X_{4}$ are orthonormal vectors. Since $\mathrm{M}$ is conformally flat we get that $\langle\widetilde{\mathcal{R}}(Z \wedge W),(Z \wedge W)\rangle$ is given only by $K_{13}+K_{24}+K_{14}+K_{23}$. We will show that if $\lambda+\mu \geq 0$ then $K_{13}+K_{24} \geq 0$ and the second term is proved in a similar manner. For that, let $X^{\prime}$ and $X^{\prime \prime}$ denote the orthogonal projection of the vector $X$ onto $\operatorname{span}\left\{e_{1}\right\}$ and $\operatorname{span}\left\{e_{2}, \ldots, e_{n}\right\}$ respectively. Let $\mathcal{R}$ denote the curvature 
operator of $M$. Since $X_{1}^{\prime} \wedge X_{3}^{\prime}=0$, using the Gauss equation we obtain

$$
\begin{aligned}
K_{13}= & \left\langle\mathcal{R}\left(X_{1} \wedge X_{3}\right), X_{1} \wedge X_{3}\right\rangle=\lambda \mu\left(\left\|X_{1}^{\prime} \wedge X_{3}^{\prime \prime}\right\|^{2}+\left\|X_{1}^{\prime \prime} \wedge X_{3}^{\prime}\right\|^{2}\right) \\
& +\lambda^{2}\left\|X_{1}^{\prime \prime} \wedge X_{3}^{\prime \prime}\right\|^{2}+2 \lambda \mu\left\langle X_{1}^{\prime} \wedge X_{3}^{\prime \prime}, X_{1}^{\prime \prime} \wedge X_{3}^{\prime}\right\rangle .
\end{aligned}
$$

Since $\left\|X_{1} \wedge X_{3}\right\|^{2}=1$, we have that

$$
\left\|X_{1}^{\prime} \wedge X_{3}^{\prime \prime}\right\|^{2}+\left\|X_{1}^{\prime \prime} \wedge X_{3}^{\prime}\right\|^{2}=1-\left\|X_{1}^{\prime \prime} \wedge X_{3}^{\prime \prime}\right\|^{2}-2\left\langle X_{1}^{\prime} \wedge X_{3}^{\prime \prime}, X_{1}^{\prime \prime} \wedge X_{3}^{\prime}\right\rangle
$$

that substituted in the above equation gives

$$
K_{13}=\lambda \mu+\lambda(\lambda-\mu)\left\|X_{1}^{\prime \prime} \wedge X_{3}^{\prime \prime}\right\|^{2} .
$$

Therefore

$$
K_{13}+K_{24}=2 \lambda \mu+\lambda(\lambda-\mu)\left(\left\|X_{1}^{\prime \prime} \wedge X_{3}^{\prime \prime}\right\|^{2}+\left\|X_{2}^{\prime \prime} \wedge X_{4}^{\prime \prime}\right\|^{2}\right) .
$$

Because we are supposing that $\lambda \geq 0$, if $\mu \geq 0$, the curvature operator is nonnegative and then the isotropic curvature is nonnegative. For the case that $\mu<0$, notice that

$$
\begin{aligned}
\left\|X_{1} \wedge X_{3}\right\|^{2}= & \left\|X_{1}^{\prime} \wedge X_{3}^{\prime \prime}\right\|^{2}+\left\|X_{1}^{\prime \prime} \wedge X_{3}^{\prime}\right\|^{2} \\
& +\left\|X_{1}^{\prime \prime} \wedge X_{3}^{\prime \prime}\right\|^{2}+2\left\langle X_{1}^{\prime} \wedge X_{3}^{\prime \prime}, X_{1}^{\prime \prime} \wedge X_{3}^{\prime}\right\rangle \\
= & \left\|X_{1}^{\prime}\right\|^{2}\left\|X_{3}^{\prime \prime}\right\|^{2}+\left\|X_{1}^{\prime \prime}\right\|^{2}\left\|X_{3}^{\prime}\right\|^{2} \\
& -2\left\langle X_{1}^{\prime}, X_{3}^{\prime}\right\rangle\left\langle X_{1}^{\prime \prime}, X_{3}^{\prime \prime}\right\rangle+\left\|X_{1}^{\prime \prime} \wedge X_{3}^{\prime \prime}\right\|^{2} .
\end{aligned}
$$

Since the vectors $X_{1}, X_{2}, X_{3}, X_{4}$ are orthonormal, we have $\left\langle X_{i}^{\prime}, X_{j}^{\prime}\right\rangle=-\left\langle X_{i}^{\prime \prime}, X_{j}^{\prime \prime}\right\rangle$ and $\left\|X_{i}^{\prime \prime}\right\|^{2}=1-\left\|X_{i}^{\prime}\right\|^{2}$. Substituting in the above equation we get

$$
1=\left\|X_{1}^{\prime}\right\|^{2}+\left\|X_{3}^{\prime}\right\|^{2}-2\left\|X_{1}^{\prime}\right\|^{2}\left\|X_{3}^{\prime}\right\|^{2}+2\left\langle X_{1}^{\prime}, X_{3}^{\prime}\right\rangle^{2}+\left\|X_{1}^{\prime \prime} \wedge X_{3}^{\prime}\right\|^{2}
$$

which in turn implies $\left\|X_{1}^{\prime \prime} \wedge X_{3}^{\prime \prime}\right\|^{2}=1-\left\|X_{1}^{\prime}\right\|^{2}-\left\|X_{3}^{\prime}\right\|^{2}$, because $X_{1}^{\prime} \wedge X_{3}^{\prime}=0$. Similar computations for $\left\|X_{2}^{\prime \prime} \wedge X_{4}^{\prime \prime}\right\|^{2}$ yield

$$
\left\|X_{1}^{\prime \prime} \wedge X_{3}^{\prime \prime}\right\|^{2}+\left\|X_{2}^{\prime \prime} \wedge X_{4}^{\prime \prime}\right\|^{2}=2-\left\|X_{1}^{\prime}\right\|^{2}-\left\|X_{2}^{\prime}\right\|^{2}-\left\|X_{3}^{\prime}\right\|^{2}-\left\|X_{4}^{\prime}\right\|^{2} .
$$

Observe that

$$
\left\|X_{1}^{\prime}\right\|^{2}+\left\|X_{2}^{\prime}\right\|^{2}+\left\|X_{3}^{\prime}\right\|^{2}+\left\|X_{4}^{\prime}\right\|^{2} \leq 1
$$

because it is the square of the norm of the orthogonal projection of the unitary vector $e_{1}$ onto $\operatorname{span}\left\{X_{1}, X_{2}, X_{3}, X_{4}\right\}$. Therefore

$$
\left\|X_{1}^{\prime \prime} \wedge X_{3}^{\prime \prime}\right\|^{2}+\left\|X_{2}^{\prime \prime} \wedge X_{4}^{\prime \prime}\right\|^{2} \geq 1
$$

Since we have $\lambda-\mu \geq 0$, the above inequality implies in (3.2) that

$$
K_{13}+K_{24} \geq 2 \lambda \mu+\lambda(\lambda-\mu)=\lambda(\lambda+\mu) \geq 0 .
$$

Now we show how to produce examples of compact hypersurfaces embedded in $\mathbf{R}^{n+1}$ that are conformally flat, have nonnegative isotropic curvature and large $\pi_{1}(M)$.

Example 3.3. Consider a hypersurface in $\mathbf{R}^{n+1}$ obtained by rotating a curve in the $\left\{x_{1}, x_{2}\right\}$-plane about the $x_{1}$-axis. Suppose that the curve is the graph of a positive function $\tau=\tau\left(x_{1}\right)$. We obtain a conformally flat hypersurface of revolution. The simple eigenvalue $\mu$ is the curvature of the generating curve (up to sign, which depends on the choice of the normal vector) and the eigenvalue $\lambda$ of multiplicity $n-1$ is the inverse of the radius $\tau\left(x_{1}\right)$ multiplied by the cosine of the angle that 
the normal makes with $x_{2}$-axis (again up to sign). If we choose the normal vector in such a way that $\lambda \geq 0$ we get

$$
\begin{gathered}
\lambda=\tau^{-1}\left(1+\left(\tau^{\prime}\right)^{2}\right)^{-1 / 2}, \\
\mu=-\tau^{\prime \prime}\left(1+\left(\tau^{\prime}\right)^{2}\right)^{-3 / 2}
\end{gathered}
$$

( the reader is referred to [7], p. 25, 12.d for the expression of $\mu$ ). Then the condition of nonnegative isotropic curvature is

$$
1+\left(\tau^{\prime}\right)^{2} \geq \tau \tau^{\prime \prime}
$$

Therefore, if $\tau\left(x_{1}\right)$ satisfies (3.4), the rotational hypersurface determined by such a function has nonnegative isotropic curvature. We use such hypersurfaces to connect to spheres, according to the Diagram 1.

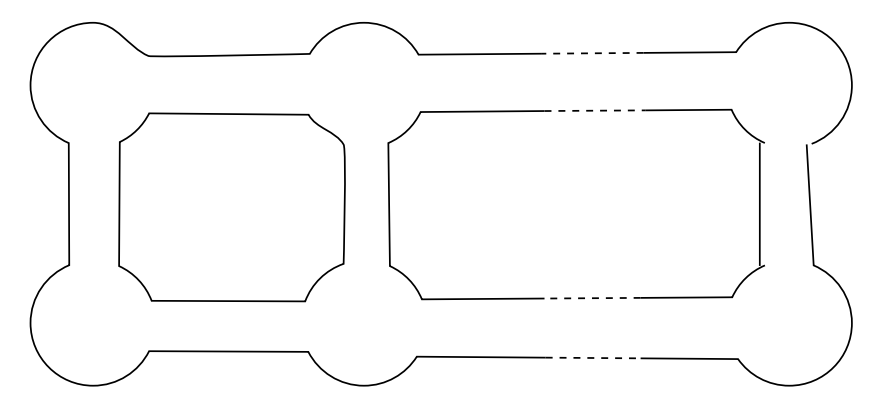

\section{DiAgRAM 1}

We obtain then compact hypersurfaces with nonnegative isotropic curvature and first Betti number any nonnegative integer. In order to complete this procedure, we claim that there exists a smooth function $\tau$ satisfying (3.4) that allows the above connections to be made smoothly. In fact, we consider the $C^{2}$-function $\tau:\left(-\frac{\sqrt{2}}{2}-\epsilon, \frac{\sqrt{2}}{2}+\epsilon\right) \rightarrow \mathbf{R}$ given by

$$
\tau(x)= \begin{cases}{\left[1-\left(x+\frac{\sqrt{2}}{2}\right)^{2}\right]^{1 / 2},} & x \leq 0 \\ -\left(x-\frac{\sqrt{2}}{2}\right)^{3}(2 \sqrt{2} x+4 / 3)+\sqrt{2} / 6, & 0 \leq x \leq \sqrt{2} / 2 \\ \sqrt{2} / 6, & x \geq \sqrt{2} / 2\end{cases}
$$

(This explicit example came out from a nice discussion with A.P. Seixas). The inequality (3.4) holds strictly for this function. Therefore for any $C^{\infty}$-function which is sufficiently $C^{2}$ close to $\tau,(3.4)$ still holds. By basic approximation, we can find a $C^{\infty}$-function which coincides with $\tau$ outside $[0, \sqrt{2} / 2]$ and is a good approximation to $\tau$ in the above sense. We observe that such a function allows us to connect the three necessary handles (according to Diagram 1 ) in the same sphere, since the tube obtained by rotating $\tau$ in $[0, \sqrt{2} / 2]$ glues in the sphere obtained by the rotation of $\tau$ for $x<0$ in a relatively small region.

Notice that the condition for nonnegative scalar curvature for conformally flat hypersurfaces is $\lambda \geq-2 \mu /(n-2)$, which is equivalent to $\lambda+\mu \geq 0$ if $n=4$, but is a stronger condition if $n \geq 5$. Therefore, if $n \geq 5$, modifying the function $\tau$, making it decrease more rapidly near zero, we can produce an example with $\lambda+\mu<0$ 
somewhere but $\lambda \geq-2 \mu /(n-2)$; i.e., conformally flat hypersurfaces of dimension $n \geq 5$ with nonnegative scalar curvature but some negative isotropic curvature.

\section{Conformally flat manifolds \\ WITH NONNEGATIVE ISOTROPIC CURVATURE}

Conditions on the isotropic curvature fit the classical Bochner technique well and this will be the basic tool to prove Theorem 2. Recall first that the Weyl tensor $W$ of a Riemannian manifold $M$ is defined by

$W(X, Y) Z=R(X, Y) Z-\langle Y, Z\rangle \gamma(X)+\langle\gamma(X), Z\rangle Y-\langle\gamma(Y), Z\rangle X+\langle X, Z\rangle \gamma(Y)$

where $\gamma: T_{x} M \rightarrow T_{x} M$ is defined by

$$
\gamma(X)=\frac{1}{n-2}\left(\operatorname{Ric}(X)-\frac{S}{2(n-1)} X\right)
$$

with $S$ denoting the scalar curvature. It is well known that if $M$ is conformally flat and $n \geq 4$ then $W$ is identically zero. Observe $W=0$ implies that for an orthonormal basis $\left\{e_{i}\right\}, i=1, \ldots, n$, which diagonalizes the symmetric Ricci operator Ric we have $\left\langle R\left(e_{i}, e_{j}\right) e_{k}, e_{m}\right\rangle=0$ whenever the set $\{i, j, k, m\}$ contains more than two elements. We will call this basis an $\mathcal{R}$-basis, since the 2 -forms $e_{i} \wedge e_{j}$ are the eigenvectors of the curvature operator $\mathcal{R}$.

Proposition 4.2. Let $M$ be a compact conformally flat manifold with nonnegative isotropic curvature. Then

a) if $\phi$ is a harmonic $k$-form, $2 \leq k \leq[n / 2]$, then $\phi$ is parallel,

b) if all isotropic curvatures are positive at some point, then $\beta_{k}(M)=0$ for $2 \leq k \leq n-2$.

Proof. We can suppose without losing generality that $M$ is orientable. Let $\phi$ be a harmonic $k$-form. Let $\Delta$ denote the Laplace-Beltrami operator and $\nabla \phi$ the covariant derivative of $\phi . \Delta \phi$ and $\nabla \phi$ satisfy the well known Weitzenböck formula for compact oriented manifolds ( see [11] )

$$
(\Delta \phi, \phi)=(\nabla \phi, \nabla \phi)+\int_{M} F(\phi) d V
$$

where (,) denotes the $L^{2}$-product with respect to the Riemannian volume density $d V$ and

$$
F(\phi)=\frac{1}{(k-1) !}\left[A-\frac{k-1}{2} B\right]
$$

with

$$
\begin{aligned}
A & =\sum_{i_{3}, \ldots, i_{k}} \sum_{r, s, u, t} \phi\left(X_{r}, X_{s}, X_{i_{3}}, \ldots, X_{i_{k}}\right) \phi\left(X_{t}, X_{r}, X_{i_{3}}, \ldots, X_{i_{k}}\right)\left\langle R\left(X_{s}, X_{u}\right) X_{u}, X_{t}\right\rangle, \\
B & =\sum_{i_{3}, \ldots, i_{k}} \sum_{r, s, u, t} \phi\left(X_{r}, X_{s}, X_{i_{3}}, \ldots, X_{i_{k}}\right) \phi\left(X_{t}, X_{u}, X_{i_{3}}, \ldots, X_{i_{k}}\right)\left\langle R\left(X_{r}, X_{s}\right) X_{u}, X_{t}\right\rangle .
\end{aligned}
$$

From the Weitzenböck formula, we see that in order to prove a), we need to show that $F(\phi) \geq 0$. We compute $A$ and $B$ for an $\mathcal{R}$-basis $\left\{e_{1}, \ldots, e_{n}\right\}$. Then we have

$$
A=\sum_{r, s} \phi\left(e_{s}, e_{r}, X_{i_{3}}, \ldots, X_{i_{k}}\right)^{2} \operatorname{Ric}\left(e_{s}, e_{s}\right)
$$




$$
B=2 \sum_{r, s} \phi\left(e_{s}, e_{r}, X_{i_{3}}, \ldots, X_{i_{k}}\right)^{2} K_{r s}
$$

where $K_{r s}$ is the sectional curvature of the plane spanned by $e_{r}$ and $e_{s}$. Therefore

$$
\begin{aligned}
& F(\phi)=\frac{1}{(k-1) !} \sum_{i_{3}, \ldots, i_{k}} \sum_{s<r} \phi\left(e_{s}, e_{r}, X_{i_{3}}, \ldots, X_{i_{k}}\right)^{2}\left(\operatorname{Ric}\left(e_{s}, e_{s}\right)+\right. \\
&\left.\operatorname{Ric}\left(e_{r}, e_{r}\right)-2(k-1) K_{r s}\right) .
\end{aligned}
$$

We will show that if $M$ is conformally flat and has nonnegative isotropic curvature, then for $2 \leq k \leq[n / 2]$ we have

$$
\left(\operatorname{Ric}\left(e_{s}, e_{s}\right)+\operatorname{Ric}\left(e_{r}, e_{r}\right)-2(k-1) K_{r s}\right) \geq 0 .
$$

Notice that

$$
\left(\operatorname{Ric}\left(e_{s}, e_{s}\right)+\operatorname{Ric}\left(e_{r}, e_{r}\right)-2 K_{r s}\right)=\sum_{i \neq r, s}\left(K_{r i}+K_{s i}\right)
$$

and then if the dimension $n$ is even, Lemma 2.2 implies that $\sum_{i \neq r, s}\left(K_{r i}+K_{s i}\right) \geq 0$. If $n$ is odd, as in the proof of Proposition 2.4, we find first $e_{m}$ such that $K_{r m}+K_{s m} \geq 0$ that together with $(2.2)$ gives

$$
\sum_{i \neq r, s}\left(K_{r i}+K_{s i}\right)=K_{r m}+K_{s m}+\sum_{i \neq r, s, m}\left(K_{r i}+K_{s i}\right) \geq 0 .
$$

Therefore, if $k=2,(4.3)$ is verified. If $k>2$ we have

$$
\operatorname{Ric}\left(e_{s}, e_{s}\right)+\operatorname{Ric}\left(e_{r}, e_{r}\right)-2(k-1) K_{r s}=\sum_{i \neq r, s}\left(K_{r i}+K_{s i}\right)-2(k-2) K_{r s}
$$

which again is nonnegative if $K_{r s} \leq 0$. Now we verify (4.3) for the case that $2<$ $k \leq[n / 2]$ and $K_{r s}>0$. If $M$ is conformally flat, we have $W=0$ and then a straightforward computation from (4.1) gives

$$
K_{r s}=\frac{1}{n-2}\left[\operatorname{Ric}\left(e_{s}, e_{s}\right)+\operatorname{Ric}\left(e_{r}, e_{r}\right)-\frac{S}{n-1}\right]
$$

which substituted in (4.3) implies

$$
\begin{aligned}
\operatorname{Ric}\left(e_{s}, e_{s}\right)+\operatorname{Ric}\left(e_{r}, e_{r}\right)-2(k-1) K_{r s} \\
\left.\quad=\frac{n-2 k}{n-2}\left[\operatorname{Ric}\left(e_{s}, e_{s}\right)+\operatorname{Ric}\left(e_{r}, e_{r}\right)\right]+\frac{2(k-1) S}{(n-2)(n-1)}\right) \\
\quad=\frac{n-2 k}{n-2}\left[2 K_{r s}+\sum_{i \neq r, s}\left(K_{r i}+K_{s i}\right)\right]+\frac{2(k-1) S}{(n-2)(n-1)} \geq 0,
\end{aligned}
$$

since nonnegative isotropic curvature implies that $S \geq 0$ and we are supposing $K_{r s}>0$.

These calculations show that if at some point all isotropic curvatures are positive then $F(\phi)>0$ at this point for every non-null $k$-form $\phi, 2 \leq k \leq[n / 2]$. Then it follows from the Weitzenböck formula that if $\Delta \phi=0$ then $\phi=0$. Therefore $\beta_{k}=0$ for $2 \leq k \leq[n / 2]$, by Hodge's Theorem. By duality we conclude then that $\beta_{k}=0$ for $2 \leq k \leq n-2$.

Before we prove Theorem 2, we mention a nice characterization of conformally flat manifolds in terms of sectional curvature given by Kulkarni in [12]. He proved 
that for $n \geq 4$, a Riemannian manifold $M^{n}$ is conformally flat if and only if for any orthonormal vectors $X_{1}, X_{2}, X_{3}, X_{4}$ we have

$$
K_{12}+K_{34}=K_{13}+K_{24}
$$

Proof of Theorem 2. Let us suppose first that $M$ is locally irreducible. Then the restricted holonomy group $G$ is also irreducible. Recall that in [3], Berger proved that if for some $x \in M, G$ acts irreducibly on $T_{x} M$, then either $M$ is locally symmetric or $G$ is one of the standard subgroups of $S O(n)$ :

$$
\begin{aligned}
& S O(n), U(m)(n=2 m), \quad S p(m) \times S p(1)(n=4 m>4), \quad \operatorname{Spin}(9)(n=16), \\
& S U(m)(n=2 m>2), \quad S p(m)(n=4 m>4), \quad G_{2}(n=7), \quad \operatorname{Spin}(7)(n=8) .
\end{aligned}
$$

If $M$ is locally symmetric, since $G$ is irreducible, the universal cover $\tilde{M}$ is an irreducible symmetric space. A result in [18] (Proposition 4.2) implies that $\tilde{M}$ is isometric to a sphere since it has nonnegative scalar curvature. Then for this case we have $\beta_{i}=0$ for $1 \leq i \leq n-1$. We now study the other cases. Berger also proved that if $G$ is one of the possibilities listed on the second line above, $M$ is Ricci flat. Since $M$ is conformally flat, this implies that the sectional curvatures vanish, contradicting the assumption that $\tilde{M}$ is irreducible. If $G=S p(m) \times S p(1)$, $M$ is an Einstein manifold ( see [4] ). For conformally flat manifolds, this implies that the sectional curvatures are constant, implying again that they are covered by a sphere. If $G=\operatorname{Spin}(9)$, it follows from [6] that $M$ is locally symmetric, and then has constant sectional curvature.

We were left with two possibilities for $G, U(m)$ or $S O(n)$. This first case implies that $\tilde{M}$ is a Kähler manifold. Conformally flat Kähler manifolds of dimension greater than 4 are flat and if $n=4, \tilde{M}$ is a product of two surfaces with mutually opposite constant curvatures (see [9], Remark 2). Either case contradicts that $\tilde{M}$ is irreducible. Then the only possibility for $G$ and therefore for the holonomy group of $M$ is $S O(n)$. Suppose that $\beta_{k}(M)>0$ for $2 \leq k \leq[n / 2]$. By the Hodge decomposition theorem, there exists one harmonic $k$-form $\phi$, that by Proposition 4.2 a), is parallel; i.e., $\phi$ is left invariant by $S O(n)$. By the holonomy principle, the existence of $\phi$ would give rise to a parallel and hence harmonic $k$-form on $S^{n}$, which is a contradiction. Therefore, by duality we conclude that $\beta_{k}(M)=0$ for $2 \leq k \leq n-2$.

Let us suppose now that $M$ is locally reducible and consider the de Rham decompositon of the universal cover $\tilde{M}=\mathbf{R}^{m} \times M_{1} \times \ldots \times M_{r}$. The $M_{i}$ 's are non flat factors and hence $\operatorname{dim} M_{i} \geq 2$. Let $Q(c)$ denote a simply connected complete manifold of constant curvature $c$. By choosing suitable orthonormal vectors and applying the criterion of Kulkarni, we get:

a) If $m \geq 2$, then $m=n$, i.e. $\tilde{M}=\mathbf{R}^{n}$,

b) If $m=1$, then $\tilde{M}=\mathbf{R} \times Q^{n-1}\left(c^{2}\right)$, in particular $\beta_{i}(M)=0$ for $2 \leq i \leq n-2$,

c) If $m=0$, then $r=2$ and $\tilde{M}=Q^{p}\left(-c^{2}\right) \times Q^{n-p}\left(c^{2}\right)$.

In the last case if $p>2$, we can take three orthonormal vectors tangent to $Q^{p}\left(-c^{2}\right)$ and a fourth tangent to $Q^{n-p}\left(c^{2}\right)$. Writing the condition of Lemma 2.2 for those vectors we get a contradiction.

Remark 4.4. In general, the vanishing of $\beta_{2}$ does not imply that the second homology group $H_{2}(M, \mathbf{Z})=0$, even for conformally flat manifolds with nonegative isotropic curvature. For example, if $M$ is the product of a circle $S^{1}$ with the real 
projective 3 -space $\mathbf{R} \mathbf{P}^{3}$ with constant curvature $1, M$ is conformally flat, has nonnegative isotropic curvature and $H_{2}(M, \mathbf{Z}) \cong \mathbf{Z}_{2}$.

Proof of Corollary 2. A theorem of Moore (see [16]) states that a compact conformally flat $n$-dimensional manifold isometrically immersed in $\mathbf{R}^{n+p}, 2 \leq p \leq n / 2-1$, has the homotopy type of a $C W$-complex with no cells in dimension $i$, for $p<i<$ $n-p$. Then, for this range of indices, $H_{i}(M, \mathbf{Z})=0$. We suppose first that $p \geq 3$. The nonnegativity of the isotropic curvature implies $\beta_{p}(M)=0$. Since there are no $p+1$-cells, $H_{p}(M, \mathbf{Z})$ is free and therefore vanishes because $\beta_{p}(M)=0$. By duality we conclude that $H_{n-p}(M, \mathbf{Z})=0$. Now, if $p=2, H_{i}(M, \mathbf{Z})=0$ for $2<i<n-2$. If $\beta_{2}(M)=0$ we obtain $H_{2}(M, \mathbf{Z})=H_{n-2}(M, \mathbf{Z})=0$, again because there are no 3-cells. Otherwise, $M$ is covered by $\mathbf{R}^{n}$ or $Q^{2}\left(-c^{2}\right) \times Q^{n-2}\left(c^{2}\right)$. The first case cannot occur since a compact flat $n$-manifold does not admit isometric immersions in codimension 2 for $n \geq 5$. In the last case, if $\pi: Q^{2}\left(-c^{2}\right) \times Q^{n-2}\left(c^{2}\right) \rightarrow M$ is an isometric covering, we consider the immersion $(f \circ \pi)$. It follows from [15] that either $(f \circ \pi)$ splits as a product of hypersurface immersions or takes complete geodesics into straight lines. But the latter case cannot occur since $\tilde{M}$ covers a compact manifold. This means that $(f \circ \pi)$ is a product of hypersurface immersions and $\left.(f \circ \pi)\right|_{Q^{2}\left(-c^{2}\right)}$ is an immersion of a hyperbolic plane in $\mathbf{R}^{3}$ which is impossible by the theorem of Hilbert.

Observe that the proof of Proposition 4.2 shows that for a 2 -form $\phi$, the nonnegativity of $F(\phi)$ in the Weitzenböck formula depends only on the existence of an $\mathcal{R}$-basis for the tangent space. Then we consider the class of manifolds with such a basis.

Definition 4.5. A Riemannian manifold is said to have pure curvature tensor if for every $x \in M$ there is an orthonormal basis $\left\{e_{1}, \ldots, e_{n}\right\}$ of the tangent space $T_{x} M$ such that $\left\langle R\left(e_{i}, e_{j}\right) e_{k}, e_{m}\right\rangle=0$ whenever the set $\{i, j, k, m\}$ contains more than two elements.

All 3-manifolds have pure curvature tensor. This class of manifolds also includes hypersurfaces of Euclidean spaces. More generally, if a manifold admits an isometric immersion into a space of constant curvature with flat normal bundle, then the manifold has pure curvature tensor. In fact, the Ricci equation implies that there is an orthonormal basis that diagonalizes simultaneously all the Weingarten operators and hence diagonalizes the second fundamental form $\alpha$. The Gauss equation shows that this basis is an $\mathcal{R}$-basis.

Theorem 4.6. Let $M$ be a compact manifold with nonnegative isotropic curvature and pure curvature tensor. Then

a) if $\phi$ is a harmonic 2-form, then $\phi$ is parallel,

b) if all isotropic curvatures are positive at some point then the second Betti number $\beta_{2}(M)=0$,

c) if $M$ is locally irreducible, then $\beta_{2}(M) \leq 1$ and, if $\beta_{2}(M)=1$ then $M$ is a simply connected Kähler manifold.

Remark 4.7. The above result was proved in [14], Theorem 2.1(b), for even dimensional manifolds and without the assumption of pure curvature tensor. We will use their result in our proof for the even-dimensional case.

Proof. The conclusions in a) and b) follow from the proof of Proposition 4.2. For c), let us consider again the restricted holonomy group $G$ of $M$ which is irreducible, 
since we are supposing that $M$ is locally irreducible. If $M$ is locally symmetric so is the universal cover $\tilde{M}$. Since it has pure curvature tensor, a theorem in [10] implies that $\tilde{M}$ has constant sectional curvature. Now $\tilde{M}$ has nonnegative scalar curvature and is irreducible. Therefore $\tilde{M}$ is isometric to a sphere which implies that $\beta_{2}(M)=0$. Note that in most of the possibilities for $G, M$ is even dimensional and the result follows from [14]. Now we consider the two remaining cases: $G$ is $S O(n)$ or $G_{2}$ if $n=7$. In the latter case, Berger also proved that $M$ is Ricci flat and the scalar curvature is identically zero. By Proposition 2.5 of [14] we conclude that $M$ is conformally flat which we already studied in Theorem 2 . Then we were left with the case that $G=S O(n)$, which gives again $\beta_{2}(M)=0$, by the holonomy principle.

\section{Codimension 2 submanifolds WITH NONNEGATIVE ISOTROPIC CURVATURE}

We start this section studying locally reducible manifolds and proving Theorem 3. The proof will follow from Lemmas 5.1, 5.2 and 5.3 below. First, we observe that Theorem 2 of Bishop in [5], implies that in this case the universal cover of $M$ is the Riemannian product $\tilde{M}=M_{1} \times M_{2}$. Let $\pi: \tilde{M} \rightarrow M$ denote the universal covering map.

Lemma 5.1. If $\operatorname{dim} M_{1}=1$ then $M$ has nonnegative sectional curvature and is diffeomorphic to the total space of sphere bundle over $S^{1}$.

Proof. We will show first that the Ricci curvature is nonnegative. Let $e_{1}$ be a unit vector tangent to $M_{1}$. Then $\operatorname{Ric}\left(e_{1}, e_{i}\right)=0$ for $i=1, \ldots, n$ and $e_{1}$ is an eigenvector of the symmetric Ricci operator Ric. Take an orthonormal basis $\left\{e_{1}, \ldots, e_{n}\right\}$ diagonalizing the operator Ric. If $\operatorname{Ric}\left(e_{i}\right)<0$ for some $2 \leq i \leq n$, there would exist $j, k \in\{2, \ldots, n\}, i \neq j, k$, such that $K_{i j}+K_{i k}<0$. Then the orthonormal vectors $e_{1}, e_{i}, e_{j}, e_{k}$ would contradict Lemma 2.2. Now, it follows from Theorem 1 in [17] that a compact $n$-dimensional manifold $M$ with nonnegative Ricci curvature isometrically immersed in $\mathbf{R}^{n+2}$ either has nonnegative sectional curvature or $\pi_{1}(M)$ is finite. The latter case cannot occur here, since $\tilde{M}$ is not compact, which implies that $\pi_{1}(M)$ is infinite. Finally, the fact that $M$ is a sphere bundle over $S^{1}$ follows from the classification of codimension two immersions of compact manifolds with nonnegative sectional curvature, given in [1] and [2].

Lemma 5.2. If $\operatorname{dim} M_{i} \geq 2$ then one of them, say $M_{1}$, has nonnegative sectional curvature and $\left.(f \circ \pi)\right|_{M_{1} \times\{p\}}$ is a convex embedding.

Proof. By a theorem of Moore (see [15]), the immersion $f \circ \pi: M_{1} \times M_{2} \rightarrow \mathbf{R}^{n+2}$ either splits as a product of two isometric immersions in codimension one or $f \circ \pi$ maps a complete geodesic onto a straight line. The latter case is not possible since $(f \circ \pi)(\tilde{M})=f(M)$ is compact, so $f$ is (locally) a product immersion. If both $M_{i}$ 's have some negative sectional curvature there exist an orthonormal basis $\left\{e_{1}, \ldots, e_{n}\right\}$ such that the first $k$ vectors are tangent to the first factor, $\alpha\left(e_{1}, e_{1}\right)=-\lambda^{2} \alpha\left(e_{2}, e_{2}\right) \neq 0, \alpha\left(e_{n-1}, e_{n-1}\right)=-\mu^{2} \alpha\left(e_{n}, e_{n}\right) \neq 0$, where $\alpha$ is the second fundamental form of the immersion. But then:

$$
\left\langle\alpha\left(e_{1}, e_{1}\right)+\alpha\left(e_{n-1}, e_{n-1}\right), \alpha\left(e_{2}, e_{2}\right)+\alpha\left(e_{n}, e_{n}\right)\right\rangle<0
$$

contradicting Proposition $2.4 \mathrm{a})$. Then we say that $M_{1}$ is the factor with nonnegative sectional curvature. Then $\left.(f \circ \pi)\right|_{M_{1} \times\{p\}}$ is a codimensional one immersion 
of a complete non flat manifold with nonnegative sectional curvature and hence a convex embedding.

Lemma 5.3. If $M_{1}$ has nonnegative sectional curvature and $\operatorname{dim}\left(M_{2}\right) \geq 3$, then $M$ has nonnegative sectional curvature and $f$ is the product of two convex embeddings.

Proof. As in the previous lemma $(f \circ \pi)$ is a product immersion. Suppose $M_{2}$ has some negative sectional curvature. Then we can find $e_{n-1}, e_{n}$ orthonormal vectors tangent to $M_{2}$ with $\alpha\left(e_{n-1}, e_{n-1}\right)=-\mu^{2} \alpha\left(e_{n}, e_{n}\right) \neq 0$. Let $e_{n-2}$ be a unit vector tangent to $M_{2}$ and orthogonal to $e_{n}, e_{n-1}$, and $e_{1}$ a vector tangent to $M_{1}$. Without loss of generality we can suppose $\alpha\left(e_{n-2}, e_{n-2}\right)=-\lambda^{2} \alpha\left(e_{n}, e_{n}\right)$. Then:

$$
\left\langle\alpha\left(e_{n-1}, e_{n-1}\right)+\alpha\left(e_{n-2}, e_{n-2}\right), \alpha\left(e_{n}, e_{n}\right)+\alpha\left(e_{1}, e_{1}\right)\right\rangle<0
$$

contradicting again Proposition 2.4. Therefore $\tilde{M}$, and hence $M$, have nonnegative sectional curvature. But then $M$ is simply connected; otherwise by the results in [1] $M$ would be covered by $\mathbf{R} \times \bar{M}$ for some irreducible manifold $\bar{M}$. Therefore, $f$ itself is a product of hypersurface immersions and each factor of $f$ is a convex embedding.

Remark 5.4. The above theorem could have been proved by an analysis of the results of Micallef and Wang on locally decomposable compact manifolds with nonnegative isotropic curvature (see [14] Theorem 3.1). However the proof would not be substantially shorter.

In the case that $M$ is locally irreducible, Bishop's theorem states that the holonomy group is $S O(n)$ or $U(2)$. In the second case $M$ is four-dimensional and we prove:

Proposition 5.5. Let $f: M \longrightarrow \mathbf{R}^{6}$ be an isometric immersion of a compact four dimensional manifold with nonnegative isotropic curvature. If the holonomy group of $M$ is $U(2)$, then the second Betti number $\beta_{2}(M)=0$ and $M$ is covered by a noncompact Kähler manifold.

Proof. Since $M$ is locally irreducible, if $\beta_{2}(M) \neq 0$ then $M$ is a simply connected Kähler manifold. From Theorem 1 in [19] we conclude that $M$ is biholomorphic to the complex projective space $\mathbf{C P}^{2}$. But such a manifold cannot be immersed in $\mathbf{R}^{6}$. Hence $\beta_{2}(M)=0$. Moreover, the above shows that $M$ cannot be simply connected; otherwise having holonomy $U(2)$, it would be a Kähler manifold. Finally, since the universal cover is Kähler, it cannot be compact, since it would have $\beta_{2}(\tilde{M}) \neq 0$ which contradicts the first part of this proof.

We do not know about the case when the holonomy is $S O(n)$. But notice that Theorem 4.6 implies in the case of pure curvature tensor, that $\beta_{2}(M)=0$. This fact together with Lemmas 5.1, 5.2, 5.3 and Proposition 5.5 give the following:

Theorem 5.6. Let $f: M^{n} \rightarrow \mathbf{R}^{n+2}, n \geq 4$, be an isometric immersion of a compact manifold $M$ with nonnegative isotropic curvature and pure curvature tensor. Then either $\beta_{2}(M)=0$ or $\tilde{M}=\Sigma^{2} \times M_{1}$, where $\Sigma$ is a surface and $M_{1}$ is diffeomorphic to a sphere. 


\section{REFERENCES}

1. Y.Baldin, F.Mercuri Isometric immersions in codimension two with nonnegative curvature, Math.Z. 173, (1980), 111-117. MR 83c:53061

2. Y.Baldin, F.Mercuri Codimension two nonorientable submanifolds with nonnegative curvature, Proc.A.M.S 103, (1988), 918-920. MR 89h:53112

3. M.Berger, Sur les groupes d'holonomie des variétés a connexion affine et des variétés riemannienes, Bull.Soc.Math.France 83, (1955), 279-310. MR 18:149a

4. M.Berger, Sur les groupes d'holonomie homogenes des variétés riemannienes, C.R. Acad.Sci.Paris serie A 262, (1966), 1316. MR 34:746

5. R.L.Bishop, The holonomy algebra of immersed manifolds of codimension two, J.Diff.Geom. 2, (1968), 347-353. MR 39:4782

6. R.B.Brown, A.Gray Riemannian manifolds with holonomy group immersed Spin(9), Differential Geometry ( in honor of K.Yano), Kinokuniya, Tokyo, (1972), 41-59. MR 48:7159

7. M do Carmo, Differential geometry of curves and surfaces, Prentice Hall, Inc., 1976. MR 52:15253

8. M.do Carmo, M.Dajczer, F.Mercuri, Compact conformally flat hypersurfaces, Trans. A.M.S. 288, (1985), 189-203. MR 86b:53052

9. A.Derdzinski, Self-dual Kähler manifolds and Einstein manifolds of dimension four, Composito Math. 49, (1983), 405-433. MR 84h:53060

10. A.Derdzinski, F.Mercuri, M.H.Noronha Manifolds with pure nonnegative curvature operator, Bol.Soc.Bras.Matem. 18, (1987), 13-22. MR 90i:53046

11. S.Gallot, D.Meyer, Opérateur de courbure et Laplacien des formes différentelles d'une variété Riemanniene, J.Math.Pures et Appl. 54, (1975), 285-304. MR 56:13128

12. R.S. Kulkarni, Curvature structure and conformal transformations, J.Diff.Geom. 4 (1970), 425-451. MR 44:2173

13. M.Micallef, J.D.Moore, Minimal two-spheres and the topology of manifolds with positive curvature on totally isotropic two-planes, Ann. of Math 127, (1988), 199-227. MR 89e:53088

14. M.Micallef, M.Y.Wang Metrics with nonnegative isotropic curvature, Duke Math.J. 72, (1993), 649-672. MR 94k:53052

15. J.D.Moore, Isometric immersions of Riemannian products, J.Diff.Geom. 5, (1971), 159-168. MR 46:6249

16. J.D.Moore, Conformally flat submanifolds of Euclidean space, Math.Ann. 225, (1977), 89-97. MR 55:4048

17. M.H.Noronha, A note on the first Betti number of submanifolds of nonnegative Ricci curvature in codimension two, Manuscr.Math.73, (1991), 335-339. MR 92h:53050

18. M.H.Noronha, Some compact conformally flat manifolds with non-negative scalar curvature, Geometriae Dedicata 47, (1993), 255-268. MR 94f:53068

19. M.H.Noronha, Self-duality and four manifolds with nonnegative curvature on totally isotropic two-planes, Michigan Math.J. 41, (1994), 3-12. MR 95e:53069

20. W.Seaman, On manifolds with non-negative curvature on totally isotropic 2-planes, Trans. A.M.S. 338, (1993), 843-855. MR 93j:53053

IMECC-UniCAmP, Universidade Estadual de Campinas, 13081-970, Campinas, SP, BRASIL

E-mail address: mercuri@ime.unicamp.br

Department of Mathematics, California State University Northridge, California 91330-8183

E-mail address: mnoronha@huey.csun.edu 